\title{
Pulsed Shortwave Therapy in Cervical Osteoarthritis: an NSAID- Controlled, Randomized Clinical Trial
}

\author{
A. Rachid El Mohammad ${ }^{1} \cdot$ Sree Koneru $^{2} \cdot$ Richard Staelin $^{3} \cdot$ Kenneth McLeod $^{4} \cdot$ Omar Tabbouche $^{1} \cdot$ lan M. Rawe $^{2}$ (D)
}

Accepted: 18 November 2020 / Published online: 6 January 2021

(C) The Author(s) 2021

\begin{abstract}
Assess treatment superiority of pulsed shortwave therapy (PSWT) against COX-2 NSAID therapy, in reducing disability and pain due to cervical osteoarthritis. Two hundred chronic pain suffers (average pain duration about 2 years) diagnosed with cervical osteoarthritis by radiological imaging were randomized into one of two treatment arms: COX-2 NSAID treatment; etoricoxib $60 \mathrm{mg}$ /day for 4 weeks; or PSWT treatment worn 24 h/day for 4 weeks. The primary outcome measure was the 4-week score on the Neck Disability Index (NDI), a 10-question assessment on a 50-point scale. Secondary outcome measures included pain (at rest and during activity) measured on a visual analog scale (VAS) of 0-100 mm, dose count of rescue pain medication (paracetamol) use, and a treatment satisfaction rating. These 4-week scores were compared across the two arms to assess superiority. After 4 weeks of treatment, subjects in both study arms reported statistically significant $(p<0.0001)$ reductions in NDI, with final scores of 11.24-NSAID and 9.34-PSWT, VAS ${ }_{\text {rest }}$, with final scores of 30.08-NSAID; 22.76-PSWT, and

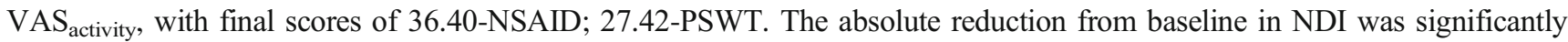
greater in the PSWT arm than NSAID arm (3.66 points; 95\% CI 2.3 to 5.02; $p<0.0001)$. Similarly, the reductions from baseline in $\mathrm{VAS}_{\text {rest }}$ and $\mathrm{VAS}_{\text {activity }}$ were significantly greater in the PSWT arm than NSAID arm (10.89 mm; $95 \%$ CI 6.90 to 14.87; $p<0.0001$; and $12.05 \mathrm{~mm}$; $95 \%$ CI 7.76 to $16.33 ; p<0.0001$, respectively). The PSWT arm used $50 \%$ less rescue pain medication. Eleven adverse effects were reported in the NSAID arm and zero in the PSWT arm. Both NSAID and PSWT treatments resulted in statistically significant improvements in quality of life (NDI) and reduction in pain (VAS) resulting from cervical osteoarthritis. However, the PSWT intervention showed superior improvements in all outcome measures when compared to the NSAID arm with no adverse effects. Clinicaltrials.gov (NCT03542955).
\end{abstract}

Keywords PSWT $\cdot$ Device $\cdot$ Osteoarthritis $\cdot$ Neck $\cdot$ Pain $\cdot$ Disability $\cdot$ Cox-2 $\cdot$ NSAID $\cdot$ Drug free

\section{Introduction}

Cervical osteoarthritis (COA), or cervical spondylosis, refers to the degeneration of the joints in the cervical region of the neck commonly arising from dehydrated/herniated vertebrae, bone spurs, and/or stiff ligaments [1]. COA is highly age dependent and is present in all adults over 40 years of age (https://bestpractice.bmj.com/topics/en-us/577/

This article is part of the Topical Collection on Medicine

Ian M. Rawe

irawe@bielcorp.com

A. Rachid El Mohammad

elmohamadrachid@yahoo.fr

Sree Koneru

skoneru@bielcorp.com

Richard Staelin

rstaelin@duke.edu

Kenneth McLeod

kmcleod@binghamton.edu
Omar Tabbouche

omartabbouche@gmail.com

1 New Mazloum Hospital, Beirut, Lebanon

2 Clinical Research Division, BioElectronics Corporation, 4539 Metropolitan Court, Frederick, MD 21704, USA

3 Duke University, Durham, NC, USA

4 Binghamton University, Binghamton, NY, USA 
epidemiology\#referencePop3). However, these degenerative changes are only weakly associated with clinical symptoms of chronic pain and stiffness in patients diagnosed with COA by radiographic imaging $[2,3]$. A similar weak association between the severity of joint degeneration and intensity of pain has been previously reported for knee osteoarthritis (KOA) $[4,5]$. In KOA, hypersensitivity in the surrounding tissues arising through central sensitization is understood to be an important factor in pain generation. Similar logic would imply that central sensitization [6] may be a contributing factor in individuals suffering with COA-related chronic pain.

While surgical interventions are recommended in more advanced stages, first-line treatment for COA ( $<6$ weeks) often involves non-steroidal anti-inflammatory drugs (NSAIDS) and physical therapy (https://bestpractice.bmj.com/topics/enus/577/epidemiology\#referencePop3). NSAIDS are widely used both as a self-administered over the counter therapy and through prescription to manage pain and inflammation resulting from osteoarthritis (OA). However, there are significant adverse effects associated with chronic use of NSAIDS, including gastrointestinal (GI) [7] and cardiovascular complications including myocardial infarction [8-10].

COX-2 specific NSAIDS (Coxibs) were developed to mitigate the incidence of GI complications associated with chronic NSAID use and have been shown to be as effective as the non-specific NSAIDS, e.g., ibuprofen, ketoprofen, and diclofenac [7]. Etoricoxib is a COX-2-specific NSAID which is orally administered [11] and well-established as a therapy for arthritic conditions including osteoarthritis of the knee [12, 13], hip [13], and rheumatoid arthritis [14]. Etoricoxib has also been shown to be an effective analgesic for acute postoperative pain [15], chronic low back pain [16], gout [17], ankylosing spondylitis $[18,19]$, and other pain conditions [20]. A daily dose of $60 \mathrm{mg}$ etoricoxib has been established as an effective therapy for relieving pain due to osteoarthritis $[12$, 18]. Where currently available, Coxibs have been shown to reduce GI-associated risks [21]; all NSAIDs have a similar risk profile with respect to cardiovascular complications [8-10, 22, 23]. The US Centers For Disease Control (CDC) acknowledges these challenges and recommends that nonpharmacological therapies be utilized as the first line of treatment against chronic pain [24].

Pulsed shortwave therapy (PSWT) is a non-invasive therapy which relies on tissue exposure to high-frequency, nonthermal electromagnetic energy [25] with the goal of providing analgesia for both acute postoperative $[26,27]$ and chronic pain [28-33]. With regard to osteoarthritis, PSWT has been demonstrated to reduce pain, improve physical functionality, and reduce the need for pharmacotherapy [28]. When used as an adjunctive therapy, PWST has also been shown to be effective in reducing pain for a number of chronic conditions within 7 days of initial use [31-33], with durability of treatment extending for at least 6 months [34]. Lack of adverse effects [33] makes PSWT especially attractive for use as a first-line treatment for COA. However, to date, no clinical study has investigated the relative effectiveness of PSWT against other first-line treatments such as NSAIDs.

The goal of this study was to investigate the effectiveness of PSWT in improving physical functionality and reducing pain in individuals diagnosed with cervical osteoarthritis in comparison to the effectiveness of NSAIDs. Specifically, we hypothesized that PSWT would provide superior performance when compared to COX-2 NSAIDs in a randomized trial of cervical osteoarthritis patients.

\section{Methods}

This single-center, parallel arm, randomized (1:1) trial was designed to test the hypothesis that PSWT is superior to NSAID as a treatment intervention for chronic pain $(\geq$ 2 months) in individuals diagnosed with COA. The study was approved by the ethics review board of New Mazloum Hospital, Tripoli, Lebanon. The procedures followed were in accordance with the ethical standards of the responsible committee on human experimentation (institutional and national) and with the Helsinki Declaration of 1975 and relevant Good Clinical Practices (GCP). The study was registered on clinicaltrials.gov (NCT03542955).

\section{Subjects}

Adult men and women who presented at the clinic of the principal investigator (Department of Neurosurgery, New Mazloum Hospital, Tripoli, Lebanon) and who were suffering neck pain and seeking treatment were screened for the study. Recruitment occurred over a period of 13 months, May 2018 through June 2019. Inclusion criteria for participation in the study included (a) a diagnosis of cervical osteoarthritis using a validated radiological imaging grading system [3]; (b) COArelated pain lasting 2 months or more; and (c) no use of prescription analgesics for at least 2 months prior to the study. Exclusion criteria included subjects who were pregnant and had osteoporosis or any neurological, muscular, hematological, or auto-immune diseases. The principal investigator fully explained the study to eligible patients including the procedures and the treatment arms; those willing to participate were enrolled into the study after providing written informed consent. Subjects who declined to be involved continued with treatment under the principal investigator. Once enrolled in the study, home visits were scheduled for the first 2 days to validate accurate use of the assigned intervention, followed by weekly in-person or telephone contact to determine patient compliance. 


\section{Treatments}

The NSAID treatment was $60 \mathrm{mg}$ etoricoxib tablets (Arcoxia ${ }^{\circledR}$ Merck, Kenilworth, NJ, USA), taken once daily, and the dose was based on prior reports of efficacy of this dose for osteoarthritis $[12,18]$ and was the standard clinically used dose which is not adjusted for body mass. Arcoxia is available in more than 80 countries worldwide, but not in the USA, where the US Food and Drug Administration (FDA) has requested additional safety and clinical testing.

The PSWT treatment was provided using a commercially available device (ActiPatch $®$, BioElectronics Corporation, Frederick, MD, USA) and was used $24 \mathrm{~h}$ daily, except when bathing. ActiPatch is an FDA class II device with indications for over-the-counter treatment of musculoskeletal pain in the USA. The device has been approved for treatment of general soft tissue pain in the EU and Canada. The device uses a loop antenna (magnetic dipole) to produce a pulsed, radio frequency magnetic field with the following signal characteristics: (1) carrier frequency of $27.12 \mathrm{MHz}$; (2) pulse width (duration) of $100 \mu \mathrm{sec}$; and (3) pulse repetition frequency (PRF) of $1 \mathrm{KHz}$. The output power of the device is approximately 10 milliWatts, into a flexible 12-cm diameter wire loop which results in a treatment area of approximately $110 \mathrm{~cm}^{2}$. This configuration results in a peak incident power density of $73 \mu \mathrm{W} / \mathrm{cm}^{2}$ when measured on the surface of the skin (measurements made using a $10 \mathrm{~cm}$ search coil terminated in a $50 \Omega$ load). The peak specific absorption rate (SAR) from the device is $0.0007 \mathrm{~W} / \mathrm{kg}$, which would result in an estimated rate of temperature rise of $2 \times 10^{-7}{ }^{\circ} \mathrm{C} / \mathrm{s}$ (correspondingly to $0.5^{\circ} \mathrm{C} /$ month in the absence of any heat loss). Given the nonthermal nature of the treatment, no temperature measurements were explicitly undertaken at the treatment site.

The device is attached over the location of pain in the cervical region with adhesive tape (Fig. 1). Subjects in both arms were provided with rescue medication (1 g paracetamol),

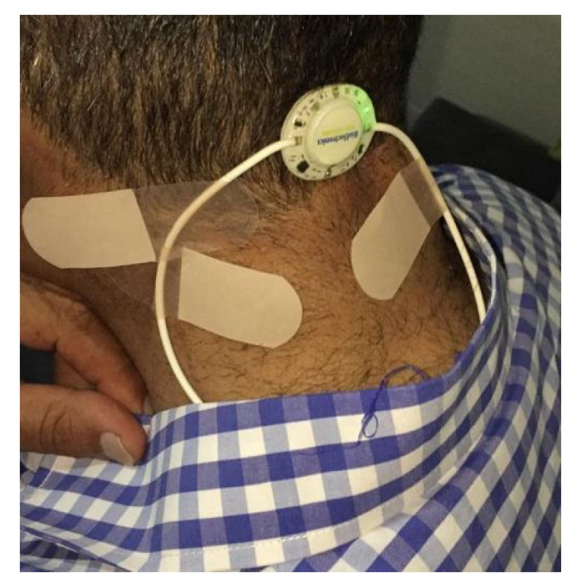

Fig. 1 Application of ActiPatch ${ }^{\circledR}$ to the cervical region of the neck. There is no paresthesia or warming sensation while the device is being used to be taken as needed in $2 \times 500 \mathrm{mg}$ tablets, with an advised maximum daily dose of $4 \mathrm{~g}$.

\section{Randomization and Treatment Arms}

Following enrollment, subjects were taken to a separate examining room and were randomized into one of the two treatment arms. Randomization was accomplished by having a designated individual randomly draw a card from a preshuffled deck of cards which initially contained 100 cards indicating NSAID and 100 cards indicating PSWT to determine the assignment for each enrolled patient. The assignment was recorded, and the drawn card was then discarded. The principal investigator was not involved in subject randomization, and did not see the patient again until the 1-month follow-up, and was therefore blinded to subject arm allocation.

\section{Measures}

Subjects provided reported pain and functional status at baseline and at the end of the 4-week study period. Initially, subjects also provided demographic information, i.e., age, gender, height, and weight (from which BMI was calculated). Functionality was measured using the Neck Disability Index (NDI), a condition-specific, functional status questionnaire with 10 items (pain, personal care, lifting, reading, headaches, concentration, work, driving, sleeping, and recreation) [35]. Each item has six possible responses, with a maximum score of 5 ( 0 being least affected, 5 being most affected), resulting in a maximum NDI score of 50 (higher scores indicate less functionality). Scores of 15-24 are classified as the individual being moderately disabled, scores of 25-34 are classified as representing severely disabled, and scores above 35 indicate complete disability. The NDI used in this study was a validated Arabic version [36]. Baseline information on pain levels at rest and during physical activity (one lateral and medial rotation of the head) were obtained using a 0-100 visual analog scale (VAS). These measures were determined by subjects marking on a $100-\mathrm{mm}$ line anchored by no pain at 0 and excruciating pain at $100 \mathrm{~mm}$. These measures were augmented by the COA imaging results.

Following 4 weeks of treatment, the subjects again provided information on functionality (via NDI) and pain levels (via VAS). In addition, rescue medication use and treatment satisfaction were recorded.

\section{Outcome Measures}

The primary outcome measures were the mean 4-week Neck Disability Index (NDI) for each arm and the difference in these two 4-week means between the two arms.

Secondary outcome measures were the mean 4-week pain scores (at rest and during physical activity) for each arm, the 4- 
week mean levels of satisfaction and medication use for each arm, and the difference of these means between the two arms.

\section{Statistical Analysis}

The size of the population needed to establish superiority in the study was calculated, a priori, by estimating a 5-point difference in average treatment effect score of the primary endpoint (NDI) between the two treatment arms. For a twotailed test with $\alpha=0.05$ and $\beta=0.2$ ( $80 \%$ power), the sample size was calculated to be 172 ( 86 in each arm). To allow for a $15 \%$ dropout rate, the total sample size for the study was set at 200 subjects.

Statistical analyses were performed using Microsoft Excel (Microsoft Corporation, Redmond, Washington) and an Excel add-on, Regressit. Analyses to determine if the treatment intervention provided significant improvement in functionality and pain relief were conducted by comparing the 4-week measures to the relevant baselines measures using two-sided
Student's $t$ tests. To test for superiority of PSWT compared to NSAIDs, 4-week scores for the relevant measures were compared using two-sided Student's $t$ tests. Since medication use and treatment satisfaction are assessed only at the end of the study, the difference across the two arms was compared using an unpaired Student's $t$ test. Analyses were performed on the intent-to-treat data set, and all tests will use $p<.05$ as the threshold for assessing statistical significance level.

\section{Results}

A total of 542 subjects are screened for eligibility for the study (Fig. 2) before the enrollment target of 200 eligible subjects was met. Twenty percent of screened subjects (108) declined to participate, while 234 did not meet the inclusion criteria. Of the 200 subjects enrolled in the study, 3 were lost to followup: 2 in the etoricoxib arm and 1 in the PSWT arm. These 3 subjects are considered dropouts but included in the statistical

Fig. 2 Subject flow diagram

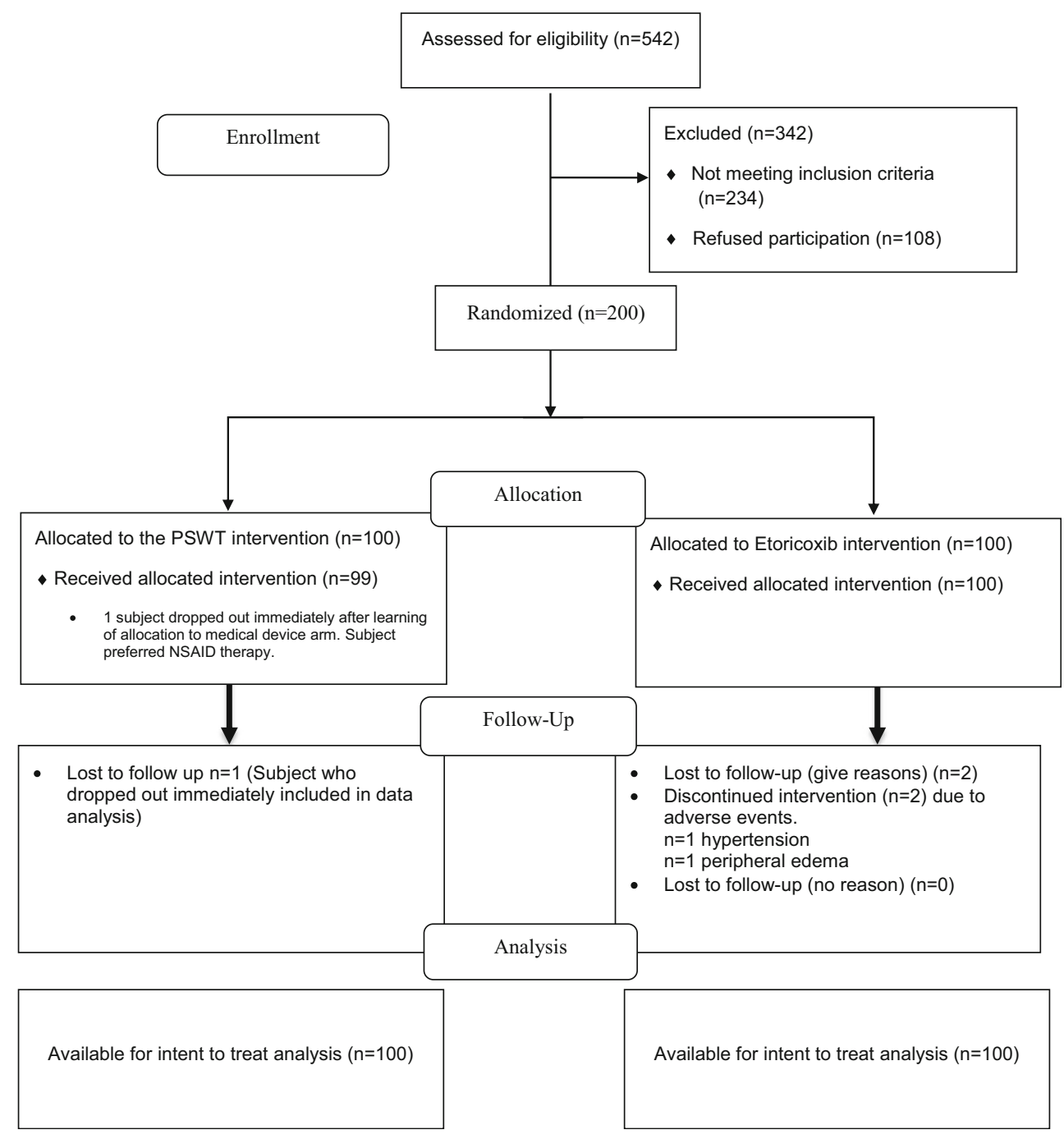


analysis by assuming no changes in any measure (Fig. 2). All remaining (197) subjects were found to be compliant with the assigned treatment regime, i.e., regularly taking prescribed dose of etoricoxib or using the PSWT device continuously except for bathing.

Participants had experienced neck pain, on average, for about 24 months and reported average baseline NDI scores of 24.5 and baseline pain scores during rest at 70.8 , and during activity at 70 VAS mm (Table 1). Statistically, significant differences were observed in baseline measures between the two arms, with the PSWT participants being older, in greater pain, and with higher disability (age: 46.4 vs 43.5 years, $p=0.04$; NDI: 25.4 vs. $23.7, p=0.0002$; $\mathrm{VAS}_{\text {rest }}: 72.6$ vs. $69, p=0.01$; VAS $_{\text {active: }} 80$ vs. $\left.76.7, p=0.01\right)$.

\section{Neck Disability Index (Functionality) and Visual Analog Scale (Pain)}

Following 4 weeks of treatment, subjects in both treatment arms reported statistically lower $(p<0.0001)$ mean scores for NDI, VAS rest $_{\text {, }}$ and $\mathrm{VAS}_{\text {active }}$ (Table 2).

To assess differential efficacy of the two interventions, we compare the endpoints (4-week means) for the three primary outcome measures (Table 3). We observed that for each outcome, the differences were statistically significant at the $p<0.0001$ level in favor of the PSWT arm. The standardized effect size on the differences is also calculated (using average of the standard deviations for the two interventions), and we observe a medium to large (0.6-0.8) standardized effect in favor of PSWT treatment for all three primary outcome measures (Fig. 3).

Finally, to control for any differences in demographic factors as well as the baseline outcome measures, we augmented our statistical analyses with ANCOVA analysis using ordinary least square regression and a dummy variable for the two arms. The dependent variables (DV) in these analyses were 4-week scores of the three primary outcome measure. The independent variables included a dummy variable associated with using the device $($ NSAID $=0 ;$ PSWT $=1$ ), subject demographics (age, BMI, gender, duration of pain, OA grade), and the baseline score of relevant outcome measure.

The predicted mean "adjusted" 4-week scores for the three measures and standard deviations of these adjusted mean are presented in the right hand columns of Table 3. Endpoint analysis again indicated that PSWT treatment resulted in lower 4-week scores (i.e., showed superiority to NSAID treatment). This analysis also demonstrated that the coefficients for both BMI and baseline relevant measures (i.e., NDI/VAS) were statistically significant, indicating that subjects with higher BMI and higher baseline NDI/VAS scores had inferior treatment efficacy at 4 weeks. No other variables were statistically significant in these analyses.

\section{Rescue Medication Use and Treatment Satisfaction}

Subjects in the NSAID arm used an average of 13.39 (10.80) doses of rescue pain medication over the 4 weeks compared to only 6.73 (9.03) dosages for the PSWT arm or a difference of 6.73 (1.41) in favor of the PSWT $(p<0.0001)$. The distribution of rescue pain medication use was markedly different with $44 \%$ of the PSWT arm using no rescue medication compared to $13 \%$ of the NSAID arm $(p<0.0001)$. Subject satisfaction was found to be greater in the PSWT with an average rating of 76.39 (19.84) compared to an average rating of 59.55 $(21.86)$ in the NSAID arm $(p<0.0001)$.

Table 1 Baseline demographic and clinical characteristics of the PSWT and NSAID arms

\begin{tabular}{|c|c|c|c|c|c|}
\hline \multicolumn{2}{|c|}{ Baseline demographic data } & $\begin{array}{l}\text { All subjects }(N=200) \\
\text { Mean (SD) }\end{array}$ & $\begin{array}{l}\text { PSWT arm }(N=100) \\
\text { Mean (SD) }\end{array}$ & $\begin{array}{l}\text { NSAID arm }(N=100) \\
\text { Mean }(\mathrm{SD})\end{array}$ & $\begin{array}{l}p \text { value of PSWT vs } \\
\text { NSAID means }\end{array}$ \\
\hline \multicolumn{2}{|l|}{ Age (year) } & $44.95(10.3)$ & $46.40(11.8)$ & $43.47(8.3)$ & 0.04 \\
\hline \multicolumn{2}{|l|}{ Height (cm) } & $164.9(7.8)$ & $165.3(7.9)$ & $164.47(7.8)$ & 0.45 \\
\hline \multicolumn{2}{|l|}{ Weight (Kg) } & $75.5(14.8)$ & $76.2(15.8)$ & $74.7(13.8)$ & 0.47 \\
\hline \multicolumn{2}{|l|}{ BMI } & $27.61(4.0)$ & $27.72(4.2)$ & $27.50(3.9)$ & 0.7 \\
\hline \multicolumn{2}{|c|}{ Disease duration (months) } & $24.13(24.1)$ & $24.4(23.5)$ & $23.9(24.8)$ & 0.88 \\
\hline \multicolumn{2}{|c|}{ Radiographic imaging COA grade } & $2.69(0.70)$ & $2.75(0.73)$ & $2.63(0.66)$ & 0.22 \\
\hline \multirow[t]{2}{*}{ Gender (\%) } & Men & $28.5 \%$ & $35 \%$ & $22 \%$ & 0.04 \\
\hline & Women & $71.5 \%$ & $65 \%$ & $78 \%$ & 0.04 \\
\hline \multicolumn{2}{|c|}{$\mathrm{VAS}_{\text {rest }}(0-100)$} & $70.78(9.9)$ & $72.57(10.93)$ & $69(8.66)$ & 0.01 \\
\hline \multicolumn{2}{|c|}{$\operatorname{VAS}_{\text {active }}(0-100)$} & $78.32(9.4)$ & $79.95(10.38)$ & $76.7(8.15)$ & 0.01 \\
\hline \multicolumn{2}{|c|}{ Neck Disability Index (NDI) (0-50) } & $24.57(3.0)$ & $25.42(3.77)$ & $23.66(2.66)$ & 0.0002 \\
\hline
\end{tabular}


Table 2 Change in outcome measures following 4 weeks of intervention

\begin{tabular}{|c|c|c|c|c|c|c|c|c|}
\hline \multirow[t]{2}{*}{ Outcome measure } & \multicolumn{4}{|c|}{ PSWT arm mean (SD) } & \multicolumn{4}{|c|}{ NSAID arm mean (SD) } \\
\hline & Baseline & 4 weeks & Mean difference & $p$ value & Baseline & 4 weeks & Mean difference & $p$ value \\
\hline NDI & $25.42(3.77)$ & $9.34(4.46)$ & $16.08(5.21)$ & $p<.0001$ & $23.66(2.66)$ & $11.24(4.68)$ & $12.42(4.54)$ & $p<.0001$ \\
\hline VAS $_{\text {rest }}$ & $72.57(10.93)$ & $22.76(12.78)$ & $49.81(15.15)$ & $p<.0001$ & $69(8.66)$ & $30.08(14.60)$ & $38.92(13.39)$ & $p<.0001$ \\
\hline VAS $_{\text {active }}$ & $79.95(10.38)$ & $27.42(14.81)$ & $52.53(15.92)$ & $p<.0001$ & $76.7(8.15)$ & $36.40(16.40)$ & $40.3(14.77)$ & $p<.0001$ \\
\hline
\end{tabular}

\section{Adverse Events}

Adverse events (AEs) associated with NSAID or PSWT use are assessed during the study period and recorded at the end of the study (Table 4). In the NSAID arm, 2 subjects reported serious AEs of peripheral edema and hypertension, following which etoricoxib treatment was ceased. There were 9 minor AEs in the NSAID arm; however, these subjects chose to continue NSAID therapy after consulting with the PI. There were no AEs reported in the PSWT arm - the sole dropout in this arm did so at the beginning of the study, citing a preference for pharmacotherapy.

\section{Dose-Response Characteristics (Post-hoc Analyses)}

The three ANCOVA analyses reported above found that treatment effectiveness (final 4-week score) was observed to be inversely proportional to subject BMI in all three cases. However, these analyses assumed the same loss of effectiveness for both treatment types as a function of BMI. The mechanism of action differs across the two arms. PSWT is a method of high-frequency magnetic stimulation, which utilizes a dipole magnetic field. The decay of the magnetic field of the PSWT antenna (along the $z$ axis perpendicular to the antenna), $B_{z}$, can be characterized as:

$$
B_{Z}=\frac{K R^{2}}{\left(Z^{2}+R^{2}\right)^{3 / 2}},
$$

where $R$ is the radius of the magnetic coil, $Z$ is the distance of the target away from the plane of the coil, and $K$ is a constant. The subject PSWT device used in the present study has a radius of $6 \mathrm{~cm}$.

The neck circumference is a useful predictor of BMI [37]. Moreover, the neck circumference can be used to calculate the diameter of the cross section of the neck and, thus, estimate the depth of the PSWT target region (cervical spine) for various BMIs. For instance, a normal neck size (i.e., for a $20 \mathrm{Kg} /$ $\mathrm{m}^{2}$ person) is about $38.1 \mathrm{~cm}$, while a $30 \mathrm{Kg} / \mathrm{m}^{2}$ person's neck size is about $43.2 \mathrm{~cm}$. This implies that the cervical spine is located at a depth of $5.6 \mathrm{~cm}$ and $6.3 \mathrm{~cm}$, respectively, from the skin for two different body sizes. Inserting these values into Eq. (1) indicates that the field intensity at the cervical spine of an individual with a BMI of 20 would be about $21 \%$ greater than the field intensity for a person with a BMI of 30 . As such, this leads us to postulate that the treatment effectiveness (analogous to dose responsiveness) of PSWT should be about $21 \%$ greater for a $20 \mathrm{Kg} / \mathrm{m}^{2}$ person compared to a $30 \mathrm{Kg} / \mathrm{m}^{2}$ person.

To test this premise, we conducted three separate univariate regression analyses for both the NSAID and PSWT arms, one for each of the outcome measures. The dependent variable (DV) in each regression was the subject's reduction in relevant outcome measure (in this case, baseline minus 4 weeks), with the independent variable being the subject's BMI. In all six of these analyses, we find that the outcome measures are negatively correlated with BMI (Table 5). Using these coefficient estimates, it is possible to estimate the change in outcome measure score for various BMIs for each of the outcome measures. For instance, we calculate that a $20 \mathrm{Kg} / \mathrm{m}^{2}$ person using

Table 3 Endpoint analysis between the two treatment arms based on the three primary measures: (1) as measured and (2) after adjusting for demographic factors and baseline scores using ANCOVA

\begin{tabular}{lllllll}
\hline Outcome measure & \multicolumn{2}{l}{ Measured 4-week score mean (SD) } & $\begin{array}{l}\text { Comparison of means } \\
(p \text { value })\end{array}$ & \multicolumn{2}{l}{$\begin{array}{l}\text { Adjusted (regression) 4-week score } \\
\text { Mean (SD) }\end{array}$} & $\begin{array}{l}\text { Comparison of means } \\
(p \text { value })\end{array}$ \\
\cline { 2 - 3 } & PSWT & NSAID & & PSWT & NSAID \\
\hline NDI & $9.34(4.46)$ & $11.24(4.68)$ & $p<.0001$ & $9.00(4.34)$ & $11.58(4.34)$ & $p<.0001$ \\
VAS $_{\text {rest }}$ & $22.76(12.78)$ & $30.08(14.60)$ & $p<.0001$ & $21.98(13.10)$ & $30.84(13.10)$ & $p<.0001$ \\
VAS $_{\text {active }}$ & $27.42(14.81)$ & $36.40(16.40)$ & $p<.0001$ & $26.44(14.82)$ & $37.35(14.82)$ & $p<.0001$ \\
\hline
\end{tabular}




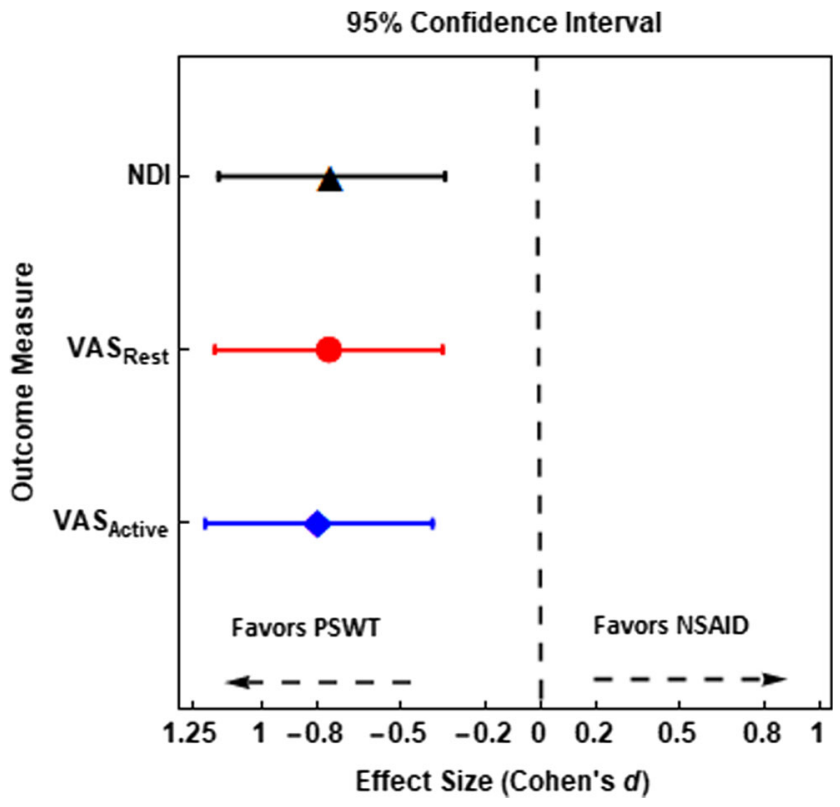

Fig. 3 Standarized effects size for the three outcome measures: NDI $0.74(95 \% \mathrm{Cl}-1.16$ to -0.34$), \mathrm{VAS}_{\text {rest }}-0.77(95 \% \mathrm{Cl}-1.17$ to -0.35$)$, and VAS $_{\text {active }}-0.796(95 \% \mathrm{Cl}-1.20$ to -0.39$)$

NSAID can expect to see a 43.69 points reduction in VAS $_{\text {active }}$ $(52.75-.453 * 20)=43.69$. However, if the person had a BMI of 30 , the reduction would only be 39.16 points $(52.75-.453 * 30)$. This implies that the effectiveness (doseresponse ratio) of the NSAID is $12 \%(=43.69 / 39.16)$ more effective when comparing a $20 \mathrm{Kg} / \mathrm{m}^{2}$ person to a $30 \mathrm{Kg} / \mathrm{m}^{2}$ person. Similarly, the figures for a person using PSWT would be a reduction of 57.85 VAS points if the person's BMI was 20 and 50.98 if the person's BMI was 30 . This implies that the device's dose-response ratio is $1.134=57.85 / 50.98$ or $13.4 \%$ more effective for the $20 \mathrm{BMI}$ person compared to the $30 \mathrm{BMI}$ person (Table 5). The dose-response ratio estimates for the NSAIDS vary from 1.07 to 1.21 , mainly because the regression coefficient for BMI was not always significant. In contrast the estimate for dose-response ratio for PSWT was very stable, ranging from 1.13 to 1.15 in line with our theoretical estimate of $23 \%$ derived earlier.

Table 4 Distribution and description of the adverse events in the two treatment arms

\begin{tabular}{lll}
\hline $\begin{array}{l}\text { Treatment } \\
\text { arm }\end{array}$ & $\begin{array}{l}\text { Adverse events/ } \\
\text { subjects }\end{array}$ & Event \\
\hline PSWT & $0 / 0$ & 0 \\
NSAID & $11 / 9$ & $\bullet$ Peripheral edema $(\mathrm{n}=1)$ \\
& & Gastric upset $(n=3)$ \\
& & Hypertension $(n=5)$ \\
& & Dysuria $(n=1)$ \\
& & Increase in serum creatinine \\
& levels \\
& & $(1.5$ to $3.5 \mathrm{mg} / \mathrm{DL})(n=1)$ \\
& & \\
\hline
\end{tabular}

Table 5 Coefficients for the univariate regression of BMI for the various outcome measures

\begin{tabular}{lll}
\hline Treatment arm & \multicolumn{2}{l}{ Regression coefficients } \\
\cline { 2 - 3 } & $\begin{array}{ll}\text { Intercept } \\
\text { Neck Disability Index (NDI) }\end{array}$ & \multicolumn{1}{l}{ BMI } \\
& & \\
& 19.21 & points $)$ \\
\hline NSAIDS & 18.99 & -.247 \\
PSWT & Visual analog scale (VAS), rest $(0-100 \mathrm{~mm})$ \\
& 47.06 & -.296 \\
NSAIDS & 70.10 & -732 \\
PSWT & Visual analog scale (VAS), activity $(0-100 \mathrm{~mm})$ \\
& 52.75 & -.453 \\
NSAIDS & 71.58 & -687 \\
PSWT & &
\end{tabular}

Also investigated was the relationship between rescue medication use and the reduction in pain. We did this using regression analysis, with the DV being rescue medication use and the independent variables being baseline demographic measures, baseline $\mathrm{VAS}_{\text {active }}$ pain level, and the change in $\mathrm{VAS}_{\text {active }}$ pain level. The only statistically significant demographic variable was age, with older subjects using more rescue medication. However, both baseline pain and pain reduction were also significant, indicating that those with higher baseline pain used more medication and importantly those experiencing the largest pain reduction used less medication.

\section{Discussion}

NSAIDS are a first-line treatment for managing pain and inflammation resulting from osteoarthritis, including COA. However, the adverse effects associated with long-term use of this treatment regime, such as GI and cardiovascular complications, have led to a search for alternative therapies. As a result, there is growing interest in the medical community to deploy non-pharmacological interventions such as medical devices for managing chronic pain [24].

This is the first study we are aware of that investigated PSWT as a primary treatment for a chronic disease state against another first-line treatment. Prior clinical studies have investigated the effectiveness of PSWT as part of multimodal therapies, both in randomized, placebo-controlled trials (RCT), and large registry studies [30, 32-34]. Notably, in a 4-week RCT on knee osteoarthritis where patients continued the use of prescribed NSAIDS (as needed), 26\% discontinued NSAID use in the PSWT arm compared to $3 \%$ in the placebo control arm [28]. Chronic pain patients (varying etiologies) who used PSWT as an adjunct therapy reported in three different registry studies a decreased reliance on 
pharmacotherapy within 7 days of using the intervention [30, $32,33]$ and also in a prospective study of 6-month duration [36].

Participants in this study reported that they were in pain for an average of about 2 years and that the pain level was currently severe and were classified at the border between being moderately and severely disabled. This latter condition was confirmed by radiological imaging where all but $7 \%$ of the samples scored OA grade 2 or higher. In addition, they were recruited after seeking specialist medical assistance. Thus, this sample of subjects could be viewed as chronic pain sufferers who were at least moderately disabled, in severe pain, and had yet to find a treatment to alleviate this pain. Following 4 weeks of treatment, subjects in both treatment arms reported statistically $(p<0.0001)$ and clinically significant reduction in disability (NDI) and pain level (VAS rest $_{\text {and }}$ VAS activity $_{\text {) }}$, with clinical significance being defined as a 7.5 point reduction (0-50 points) for NDI [38] and $20 \mathrm{~mm}$ reduction $(100 \mathrm{~mm}$ scale) for VAS [39].

More specifically, following 4 weeks of intervention, the average patient was no longer classified as being even moderately disabled with the pain levels in the PSWT arm in the mild range and in the low-moderate range for the NSAID arm. Importantly, the PSWT arm reported statistically greater reductions for all three of these measures compared to the NSAID arm. The PSWT intervention group also reported using approximately $50 \%$ less rescue medication compared to the NSAID arm $(p<.0001)$. Moreover, $44 \%$ of the PSWT arm used no rescue medication compared to $13 \%$ of the NSAID arm subjects. Finally, the PSWT group reported significantly greater overall satisfaction.

Providing non-pharmacological alternatives to NSAIDs in managing COA could also have economic advantages. One recent study indicated that up to $31 \%$ of costs in managing arthritis patients can be attributed to NSAID-related complications [40]. Another study indicated that more than 100,000 patients are hospitalized due to NSAID-related GI adverse effects each year, with direct costs between $\$ 1800$ and $\$ 8500$ per patient per hospitalization. In the elderly alone, NSAID-related GI adverse effects were reported to cost more than $\$ 4$ billion a year in the USA [41]. Similarly, in the UK, the cost to each clinical commissioning groups (CCGs) due to NSAID-related GI adverse effects averages $£ 433,000$ or an estimated total of $£ 251$ million to the UK's National Health Service [42].

In this study, 9 subjects (9\%) in the NSAID arm reported adverse events, with 2 subjects withdrawing from the study due to adverse events. No adverse effects were reported in the PSWT arm. Moreover, no serious adverse side effects associated with the use of PSWT have been reported across a variety of chronic pain etiologies for study periods up to 6 months [27-30,32-34]. Minor adverse effects that have been reported were related to method of attachment to the skin (taping, application by wraps, etc.). While the data used to gauge the possible magnitude of adverse effects associated with PSWT therapy are relatively small (hundreds of patients from clinical trials and tens of thousands from registry studies), the method of application and mechanism of action associated with PSWT indicate that the risk of adverse side effects is low. This is a major advantage when compared to pharmacological treatments such as NSAID which have been shown to dramatically increase the cost of treatment of chronic disease such as arthritis, due to the significant number of adverse side effects.

The mechanism of action of PSWT therapy is believed to be magnetic neuromodulation [43], although the precise pain signaling pathways involved are still being elucidated. However, the observed influence of BMI on efficacy may serve to help identify the specific responding tissues to the stimulation. The observed decrease in efficacy of the PSWT therapy as a function of body size makes it possible to calculate the depth below the skin where the PSWT is working. This depth analysis leads to the suggestions that the neuromuscular tissues running along the spine are the target tissues for this novel therapy.

\section{Limitations}

Lack of a placebo control group is a limitation of this study, but it is now being acknowledged that the placebo effect is built-in to any given treatment [44]. Prior chronic pain placebo-controlled trials using this device reported modest placebo effects $[28,29]$, and the device performs better than placebo in acute pain studies $[26,27,45]$ suggesting that the efficacy of the device is not only driven solely by the placebo effect. Furthermore, in this study, we find the reduction in efficacy of both treatments being associated with the subject's BMI, especially for the PSWT arm, to be strong evidence of device efficacy since this association should be independent of the placebo effect, and instead be due to the proposed mechanisms of action associated with both treatments. However further study is needed to confirm the magnitude of the placebo effect in COA as well as determine the durability of treatment. In addition, the study duration was relatively short, providing only 4 weeks of treatment. While a recent study demonstrated the durability of PSWT treatment effectiveness over a 6-month period [46], subjects in that study utilized PSWT treatment as part of multimodal therapy. As such, longer term studies are warranted.

\section{Conclusion}

Both NSAID therapy and neuromodulation therapy using PSWT resulted in statistically and clinically important reductions in pain level and improvement in functionality associated with cervical osteoarthritis, when used for 4 weeks. 
However, the PSWT intervention demonstrated superior improvements in all outcome measures when compared to the etoricoxib therapy arm, including patient satisfaction rating and decreased use of rescue pain medication. These results suggest that neuromodulation using PSWT may be a superior pain treatment option, when compared to COX-2 NSAIDS for neck osteoarthritis, and as well, represents a non-invasive, non-pharmacologic treatment option.

Supplementary Information The online version contains supplementary material available at https://doi.org/10.1007/s42399-020-00652-y.

Acknowledgments The authors would like to thank the late Andrew J Whelan for his support, passion, and guidance.

Authors' Contributions RM and OT implemented the study and oversaw data collection. IR and OT contributed to the design of the study. RS and SK contributed to the statistical analysis of the data. IR, RS, KM, and SK provided data interpretation as well as contributing to the drafting and editing of the paper for intellectual content. IR takes responsibility for the integrity of the study from beginning to finalization of the completed article.

Funding The study was funded by Bioelectronics Corp.

\section{Compliance with Ethical Standards}

Conflict of Interest IR and SK are paid employees of BioElectronics Corporatoion. RS and KM are consultants for Bioelectronics Corporation. OT is a distributor of BioElectronics medical devices. RM received a fee for participating in the study.

Role of Funding Source BioElectronics Corp funded the study, but had no role in the execution of the study, patient recruitment or data collection. BioElectronics employees along with BioElectronics consultants were involved in data analysis and in the writing of the manuscript.

Open Access This article is licensed under a Creative Commons Attribution 4.0 International License, which permits use, sharing, adaptation, distribution and reproduction in any medium or format, as long as you give appropriate credit to the original author(s) and the source, provide a link to the Creative Commons licence, and indicate if changes were made. The images or other third party material in this article are included in the article's Creative Commons licence, unless indicated otherwise in a credit line to the material. If material is not included in the article's Creative Commons licence and your intended use is not permitted by statutory regulation or exceeds the permitted use, you will need to obtain permission directly from the copyright holder. To view a copy of this licence, visit http://creativecommons.org/licenses/by/4.0/.

\section{References}

1. McCormack BM, Weinstein PR. Cervical spondylosis. An update. West J Med. 1996;165(1-2):43-51.

2. Bogduk N. The anatomy and pathophysiology of neck pain. Phys Med Rehabil Clin N Am. 2003;14(3):455-72 v.

3. Rudy IS, et al. The correlation of radiographic findings and patient symptomatology in cervical degenerative joint disease: a crosssectional study. Chiropr Man Therap. 2015;23:9.
4. Campbell CM, Buenaver LF, Finan P, Bounds SC, Redding M, McCauley L, et al. Sleep, pain catastrophizing, and central sensitization in knee osteoarthritis patients with and without insomnia. Arthritis Care Res (Hoboken). 2015;67(10):1387-96.

5. Lluch E, Torres R, Nijs J, van Oosterwijck J. Evidence for central sensitization in patients with osteoarthritis pain: a systematic literature review. Eur J Pain. 2014;18(10):1367-75.

6. Woolf CJ. Central sensitization: implications for the diagnosis and treatment of pain. Pain. 2011;152(3 Suppl):S2-15.

7. Sostres C, Gargallo CJ, Arroyo MT, Lanas A. Adverse effects of non-steroidal anti-inflammatory drugs (NSAIDs, aspirin and coxibs) on upper gastrointestinal tract. Best Pract Res Clin Gastroenterol. 2010;24(2):121-32.

8. Walker C, Biasucci LM. Cardiovascular safety of non-steroidal anti-inflammatory drugs revisited. Postgrad Med. 2018;130(1): 55-71.

9. Bally M, Beauchamp ME, Abrahamowicz M, Nadeau L, Brophy JM. Risk of acute myocardial infarction with real-world NSAIDs depends on dose and timing of exposure. Pharmacoepidemiol Drug Saf. 2018;27(1):69-77.

10. Bally M, et al. Risk of acute myocardial infarction with NSAIDs in real world use: bayesian meta-analysis of individual patient data. BMJ. 2017;357:j1909.

11. Takemoto JK, Reynolds JK, Remsberg CM, Vega-Villa KR, Davies NM. Clinical pharmacokinetic and pharmacodynamic profile of etoricoxib. Clin Pharmacokinet. 2008:47(11):703-20.

12. Moss P, Benson HAE, Will R, Wright A. Fourteen days of etoricoxib $60 \mathrm{mg}$ improves pain, hyperalgesia and physical function in individuals with knee osteoarthritis: a randomized controlled trial. Osteoarthr Cartil. 2017;25(11):1781-91.

13. da Costa BR, Reichenbach S, Keller N, Nartey L, Wandel S, Jüni P, et al. Effectiveness of non-steroidal anti-inflammatory drugs for the treatment of pain in knee and hip osteoarthritis: a network metaanalysis. Lancet. 2017;390(10090):e21-33.

14. Bickham K, Kivitz AJ, Mehta A, Frontera N, Shah S, Stryszak P, et al. Evaluation of two doses of etoricoxib, a COX-2 selective nonsteroidal anti-inflammatory drug (NSAID), in the treatment of rheumatoid arthritis in a double-blind, randomized controlled trial. BMC Musculoskelet Disord. 2016;17:331.

15. Clarke R, Derry S, Moore RA. Single dose oral etoricoxib for acute postoperative pain in adults. Cochrane Database Syst Rev. 2014;5: CD004309.

16. Zerbini C, Ozturk ZE, Grifka J, Maini M, Nilganuwong S, Morales $\mathrm{R}$, et al. Efficacy of etoricoxib $60 \mathrm{mg} /$ day and diclofenac $150 \mathrm{mg} /$ day in reduction of pain and disability in patients with chronic low back pain: results of a 4-week, multinational, randomized, doubleblind study. Curr Med Res Opin. 2005;21(12):2037-49.

17. Zhang S, Zhang Y, Liu P, Zhang W, Ma JL, Wang J. Efficacy and safety of etoricoxib compared with NSAIDs in acute gout: a systematic review and a meta-analysis. Clin Rheumatol. 2016;35(1): 151-8.

18. Balazcs E, Sieper J, Bickham K, Mehta A, Frontera N, Stryszak P, et al. A randomized, clinical trial to assess the relative efficacy and tolerability of two doses of etoricoxib versus naproxen in patients with ankylosing spondylitis. BMC Musculoskelet Disord. 2016;17(1):426

19. Gratacos J, et al. Etoricoxib in ankylosing spondylitis: is there a role for active patients refractory to traditional NSAIDs? Clin Exp Rheumatol. 2016;34(1):94-9.

20. Matsumoto AK, Cavanaugh PF Jr. Etoricoxib. Drugs Today (Barc). 2004;40(5):395-414.

21. Feng X, Tian M, Zhang W, Mei H. Gastrointestinal safety of etoricoxib in osteoarthritis and rheumatoid arthritis: a meta-analysis. PLoS One. 2018;13(1):e0190798.

22. Cannon CP, Curtis SP, FitzGerald GA, Krum H, Kaur A, Bolognese JA, et al. Cardiovascular outcomes with etoricoxib 
and diclofenac in patients with osteoarthritis and rheumatoid arthritis in the multinational etoricoxib and diclofenac arthritis long-term (MEDAL) programme: a randomised comparison. Lancet. 2006;368(9549):1771-81.

23. Pepine CJ, Gurbel PA. Cardiovascular safety of NSAIDs: additional insights after PRECISION and point of view. Clin Cardiol. 2017;40(12):1352-6.

24. Dowell D, Haegerich TM, Chou R. 2016. https://www.cdc.gov/ $\mathrm{mmwr} /$ volumes $/ 65 / \mathrm{rr} / \mathrm{rr} 6501 \mathrm{e} 1 . \mathrm{htm}$ ?CDC_AA_refVal=https\% $3 \mathrm{~A} \% 2 \mathrm{~F} \% 2 \mathrm{Fwww} . \mathrm{cdc}$.gov\%2Fmmwr\%2Fvolumes $\% 2 \mathrm{~F} 65 \%$ 2Frr\%2Frr6501e1er.htm\#contribAff.

25. Koneru SN, Westgate CR, McLeod KJ. Rectification of RF fields in load dependent coupled systems: application to non-invasive electroceuticals. J Biomed Sci Eng. 2016;9:112-21.

26. Khooshideh M, Latifi Rostami SS, Sheikh M, Ghorbani Yekta B, Shahriari A. Pulsed electromagnetic fields for postsurgical pain management in women undergoing cesarean section: a randomized, double-blind, Placebo-controlled trial. Clin J Pain. 2017;33(2):1427.

27. Rawe IM, Lowenstein A, Barcelo CR, Genecov DG. Control of postoperative pain with a wearable continuously operating pulsed radiofrequency energy device: a preliminary study. Aesthet Plast Surg. 2012;36(2):458-63.

28. Bagnato GL, Miceli G, Marino N, Sciortino D, Bagnato GF. Pulsed electromagnetic fields in knee osteoarthritis: a double blind, placebo-controlled, randomized clinical trial. Rheumatology (Oxford). 2016;55(4):755-62.

29. Brook J, Dauphinee DM, Korpinen J, Rawe IM. Pulsed radiofrequency electromagnetic field therapy: a potential novel treatment of plantar fasciitis. J Foot Ankle Surg. 2012;51(3):312-6.

30. Koneru S, Staelin R, Rawe IM. Chronic pain intervention using pulsed shortwave therapy: the relationship between pain demographics and central sensitization inventory (CSI). Pain Manag. 2019;9(3):283-96.

31. Rawe I. A registry study to assess the durability of ActiPatch ${ }^{\circledR}$ - a novel OTC neuromodulation therapy for chronic pain. Br J Pain. 2016;10(2 Suppl 1):62-3.

32. Rawe IM, Kotak DC. A UK registry study of the effectiveness of a new over-the-counter chronic pain therapy. Pain Manag. 2015;5(6): 413-23.

33. Staelin R, Koneru SN, Rawe IM. An over-the-counter central sensitization therapy: a chronic back pain registry study of pain relief, medication use and their adverse effects. Pain Manag. 2017;7(2): 99-111.
34. Staelin R, Koneru SN, Rawe IM. A prospective six-month study of chronic pain sufferers: a novel OTC neuromodulation therapy. Pain Res Manag. 2019;2019:3154194.

35. Saltychev M, Mattie R, McCormick Z, Laimi K. Psychometric properties of the neck disability index amongst patients with chronic neck pain using item response theory. Disabil Rehabil. 2018;40(18):2116-21.

36. Shaheen AA, Omar MT, Vernon H. Cross-cultural adaptation, reliability, and validity of the Arabic version of neck disability index in patients with neck pain. Spine (Phila Pa 1976). 2013;38(10): E609-15.

37. Hingorjo MR, Qureshi MA, Mehdi A. Neck circumference as a useful marker of obesity: a comparison with body mass index and waist circumference. J Pak Med Assoc. 2012;62(1):36-40.

38. Dennison BS, Leal MH. Chapter 7 - mechanical neck pain. In: Neck and arm pain syndromes. 1st ed: Churchill Livingstone; 2011. p. 94-111.

39. Katz NP, Paillard FC, Ekman E. Determining the clinical importance of treatment benefits for interventions for painful orthopedic conditions. J Orthop Surg Res. 2015;10:24.

40. Laine L. Gastrointestinal effects of NSAIDs and coxibs. J Pain Symptom Manag. 2003;25(2 Suppl):S32-40.

41. Bidaut-Russell M, Gabriel SE. Adverse gastrointestinal effects of NSAIDs: consequences and costs. Best Pract Res Clin Gastroenterol. 2001;15(5):739-53.

42. Moore RA, Philips CJ. Cost of NSAID adverse effects to the UK National Health Service. J Med Econ. 2008;2(1-4):45-55.

43. Koneru SN, McLeod KJ (2017) Increasing cardiac output in subjects with orthostatic hypotension: neuromodulation using pulsed shortwave therapy. Transactions of The International Neuromodulation Society, (The International Neuromodulation Society).

44. de Campos GC. Placebo effect in osteoarthritis: why not use it to our advantage? World J Orthop. 2015;6(5):416-20.

45. Stocchero M, Gobbato L, de Biagi M, Bressan E, Sivolella S. Pulsed electromagnetic fields for postoperative pain: a randomized controlled clinical trial in patients undergoing mandibular third molar extraction. Oral Surg Oral Med Oral Pathol Oral Radiol. 2015;119(3):293-300.

46. Staelin R, Koneru S, Rawe I. A prospective six-month study of chronic pain sufferers: a novel OTC neuromodulation therapy. Pain Res Manag. 2019;2019:1-11.

Publisher's Note Springer Nature remains neutral with regard to jurisdictional claims in published maps and institutional affiliations. 